\title{
Unusual arterial supply of the segment IV with interlobar bridge and right replaced hepatic artery: a case report
}

\author{
M. Tiryakioğlu' ${ }^{1}$, T. Koç ${ }^{2}$, N.S. İlgi ${ }^{1}$ \\ ${ }^{1}$ Department of Anatomy, Faculty of Medicine, Near East University, Nicosia, Cyprus \\ ${ }^{2}$ Department of Anatomy, Faculty of Medicine, Mersin University, Mersin, Turkey
}

[Received: 21 May 2018; Accepted: 29 July 2018]

\begin{abstract}
A replaced right hepatic artery (RHA) arising from the superior mesenteric artery and an interlobar parenchymal bridge over the sagittal fissure have been observed on a 64-year-old formalin-fixed male cadaver in the anatomy laboratory. As we had followed a detailed segmental anatomy, we encountered an arterial distribution of segment IV featuring a different pattern from the literature so far. According to our observations, the segment I is supplied by both left (LHA) and middle (MHA) hepatic arteries; the segments II and III are supplied by the LHA while the segment IV is supplied by both the MHA and replaced RHA. The segments V-VIII are supplied only by the replaced RHA.

The case emphasizes the importance of arterial variations of liver in terms of the surgical procedures during the liver transplantation, hepatic resections, hepatic tumours, and etc. Our discussion focuses to the arterial supply of the segment IV and possible complications it may cause during/after the liver operations. (Folia Morphol 2019; 78, 2: 450-454)
\end{abstract}

Key words: replaced right hepatic artery, liver, anatomy, cadaver

\section{INTRODUCTION}

During the $8^{\text {th }}$ and $10^{\text {th }}$ gestational weeks, hepatic arteries start to be visible in the hepatic hilum and take their last form in the liver parenchyma in the end of $10^{\text {th }}$ gestational week [7]. According to Couinaud classification, three main lobes are described during the embryological stage as the lateral (segment II), medial-anterior (segments III, IV, V, and VIII), and the posterior lobe (segments VI and VII). All of the lobes supplied by an embryonic artery of its own during the development of hepatic formations which are named as the embryonic left, medial, and right hepatic arteries [9]. Development of the intrahepatic arterial formation is parallel to the order of the portal and biliary systems [7]. In the adult liver, the right hepatic artery (RHA) supplies the four segments: $\mathrm{V}, \mathrm{VI}, \mathrm{VII}$, and VIII; the left hepatic artery (LHA) supplies the lateral superior and lateral inferior segments: II and III, and the middle hepatic artery (MHA) supplies the medial superior and medial inferior segments: segment IV. The caudate lobe (segment I) is supplied by both RHA and LHA [5].

In the literature, hepatic arteries have many variations in terms of their origins. Two main classifications have been described so far: replaced $[2,4,6,12$, $16]$ and accessory hepatic arteries $[11,12,16]$. The accessory hepatic artery is present in addition to the main artery of the right and/or left lobes $[2,16]$ while the replaced hepatic artery originates from another artery instead of the proper hepatic artery (usually

Address for correspondence: Dr. M. Tiryakioğlu, Near East University, Faculty of Medicine, Department of Anatomy, Nicosia, Cyprus, e-mail: mehtap.tiryakioglu@neu.edu.tr 
from the superior mesenteric artery or the coeliac trunk) $[2,12,16]$. The vascular segmental distribution of liver has critical importance in liver transplantation $[10,14]$; particularly the segment IV of liver is at greater risk for development of ischaemia, necrosis, and hepatic artery thrombosis as a post-transplant/ post-operational complication [1, 14].

In this particular report, we aimed to emphasize the arterial supply of segment IV and replaced RHA (rRHA) with their clinical and morphometric features besides drawing attention to an interlobar parenchymal bridge running between the right and left lobes of liver on its visceral surface.

\section{CASE REPORT}

A rRHA (Figs. 1, 2) and an interlobar parenchymal bridge over the sagittal fissure (Fig. 3) have been encountered during a routine abdominal dissection of 64-year-old male cadaver in the Anatomy Department of the Near East University, Faculty of Medicine. The cadaver identification and medical history did not show any of the diseases, abdominal surgeries, or cirrhosis.

In our case the common hepatic artery (CHA) originated from the coeliac trunk (CT) and passed anterior to the portal vein (PV) before it gave off the LHA and the gastroduodenal artery (GA) (Figs. 1, 2). Its length and diameter were measured as $22 \mathrm{~mm}$ and $4 \mathrm{~mm}$, respectively.

The LHA arose from the CHA with a diameter of $3 \mathrm{~mm}$ and run anterior to the PV. It gave off the MHA $4 \mathrm{~mm}$ distal to its origin and ramified into three terminal branches. The terminal branches of the LHA entered into the segments II (dia $=2 \mathrm{~mm}$ ) and III (dia $=2 \mathrm{~mm}$ ) of the left lobe. The LHA also gave off small branches (dia $=0.8 \mathrm{~mm}$ ) to the caudate lobe before it gave off the terminal branches. The length and diameter of the MHA were $41 \mathrm{~mm}$ and $2 \mathrm{~mm}$, respectively. The MHA gave five branches off: one of them ran through the umbilical fissure; three of them entered to the segment IV, and one branch entered to the caudate lobe (segment I) (Figs. 1, 2).

The rRHA occurred as arising $20 \mathrm{~mm}$ distal to the origin of the SMA, posterior to the PV, and coursed lateral to the PV. The length and diameter of the rRHA were measured as $77 \mathrm{~mm}$ and $3.5 \mathrm{~mm}$, respectively. It divided into two thick branches in Calot's triangle; one of these branches (with dia $=2 \mathrm{~mm}$ ) entered into the liver segments V-VIII while the other one gave off the cystic artery (dia $=1.5 \mathrm{~mm}$ ) and entered into the segment IV (dia $=2 \mathrm{~mm}$ ) (Figs. 1, 2). The cystic

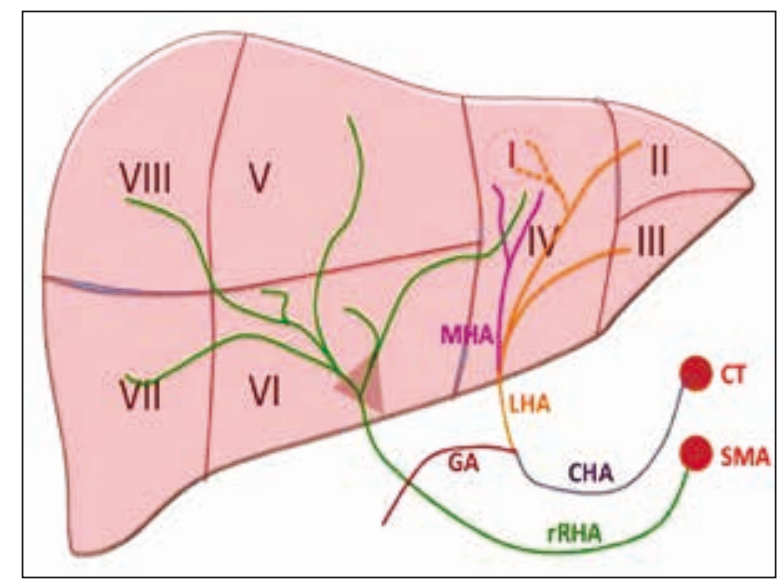

Figure 1. Schematic drawing showing the segmental distribution of hepatic arteries; I-VIII — segments of liver; CHA — common hepatic artery; CT — coeliac trunk; GA — gastroduodenal artery; LHA — left hepatic artery; MHA — middle hepatic artery; rRHA replaced right hepatic artery; SMA - superior mesenteric artery; Triangle — Calot's triangle.

artery separated into two branches on its course; one ran superiorly and supplied the anterior surface of the gallbladder and it ended in the right lobe while the other branch supplied the cystic duct and reached the gallbladder, inferiorly (Fig. 1). In our case the arterial supply to the segment IV was showing a significant difference as it is supplied by the branches from both MHA and the rRHA that was originated from the superior mesenteric artery.

In addition to the deviation on the arterial supply of the segment IV, there was an interlobar parenchymal bridge running between the right and left hepatic lobes on its visceral surface right behind the sagittal fissure. It was $2 \mathrm{~cm}$ long and $2 \mathrm{~cm}$ inferior to porta hepatis as appeared on Figure 3. The bridge can also be described like it was where the sagittal fissure missing or became a tunnel with an uninterrupted parenchyma in between the right and left lobes. We could not trace the arterial structure within the bridge, but when we split the tissue parallel to the sagittal fissure, we observed that the round ligament of liver was located right in front of it.

\section{DISCUSSION}

In the literature, 10 variant subtypes of the hepatic arterial system were reported by Michels [16] and 6 subtypes were described by Hiatt et al. [12]. Our case is in accordance with both Michels's and Hiatt's classification as type III (as the RHA is being originated from the superior mesenteric artery). 


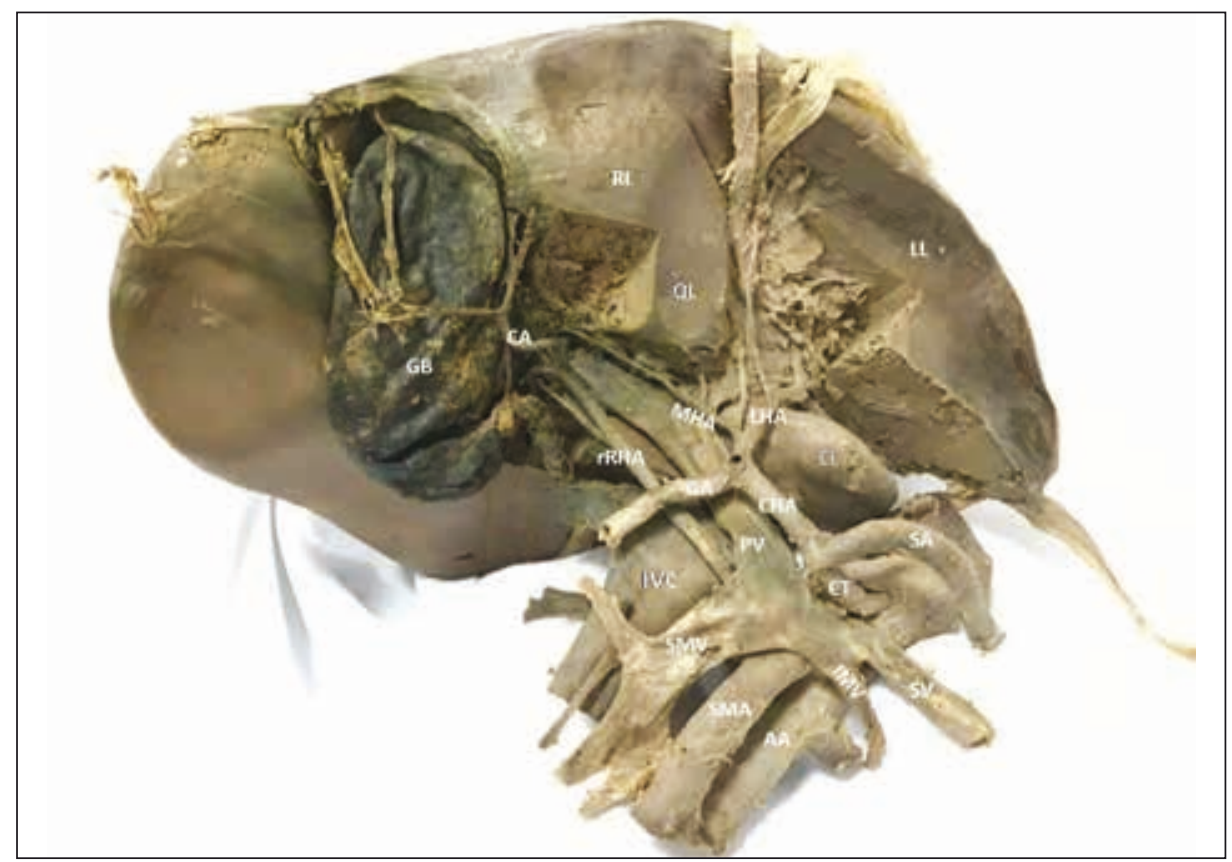

Figure 2. Visceral surface of liver showing the hepatic arteries; AA — abdominal aorta; CA — cystic artery; CHA — common hepatic artery; $\mathrm{CL}$ — caudate lobe; CT — coeliac trunk; GA — gastroduodenal artery; GB — gallbladder; IMV — inferior mesenteric vein; IVC — inferior vena cava; LHA — left hepatic artery; LL — left lobe; MHA — middle hepatic artery; PV — portal vein; $\mathrm{OL}$ — quadrate lobe; RL — right lobe; rRHA — replaced right hepatic artery; SA — splenic artery; SMA — superior mesenteric artery; SMV — superior mesenteric vein; SV — splenic vein.

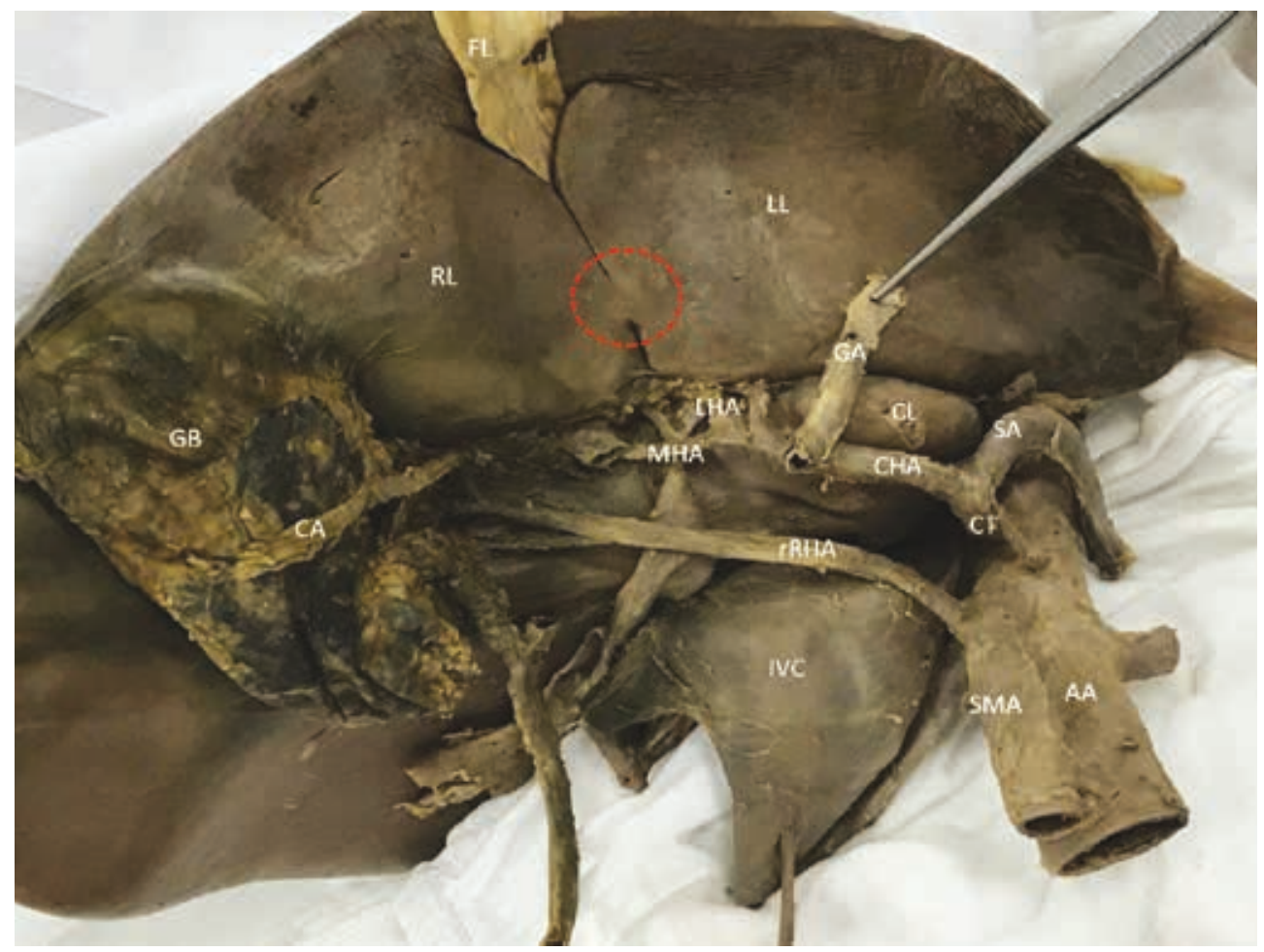

Figure 3. Photograph showing the interlobar bridge over the sagittal fissure on the visceral surface of liver; $A A-$ abdominal aorta; $C A-$ cystic artery; CHA — common hepatic artery; $\mathrm{CL}$ — caudate lobe; CT — coeliac trunk; FL — falciform ligament; GA — gastroduodenal artery; GB — gallbladder; IVC — inferior vena cava; LHA — left hepatic artery; LL — left lobe; MHA — middle hepatic artery; RL — right lobe; rRHA — replaced right hepatic artery; SA — splenic artery; SMA — superior mesenteric artery; red circular line showing the interlobar bridge. 
The vascular segmental distribution of liver has critical importance during the liver transplantations $[1,5,10,21]$; particularly the segment IV is at greater risk as it may cause a hepatic artery thrombosis as a post-transplant complication [10, 13, 14]. The MHA generally supplies the segment IV [10]. In the literature, researchers reported that the MHA originated in about equal proportions from the RHA and the LHA $[12,21]$ whereas some studies showed that the MHA originates from the LHA more often than from the RHA [17, 20]. Wang et al. [21] reported that MHA could originate from the RHA in the presence of a replaced LHA as well as from the LHA in the presence of rRHA as seen in our case. Recent studies show that the MHA diverged from the LHA in 54-61.5\% of the cases while originated from the RHA in $27.5-34 \%$ of the cases [17, 20], also the MHA originated from both the RHA and LHA in 5.5\% of the cases [17]. The origin of the MHA may also be very important for some of the clinical practices such as a right lobe resection and/or pancreaticoduodenectomy operations. Interestingly, MHA originated from the LHA could also originate from the RHA, especially in the presence of a replaced LHA according to Azoulay et al. [3].

In our case, both rRHA and MHA occurred as supplying the segment IV indicating a possible increased risk of intraoperative injury to the MHA during right/ left lobe living donor liver transplantation. Furthermore, complications of surgery such as bleeding would be eliminated by acknowledging the relationship of rRHA with the PV [19]. In our case, rRHA coursed posterior to the PV first and then it coursed laterally and superiorly as it goes farther from it.

As for the parenchymal interlobar bridge, it seemed to be a continuous parenchymal tissue jumping over the sagittal fissure in between the right and left lobes creating a gross anatomical variation, yet we could not trace its vascular structure. The sagittal fissure partially has become a $2 \mathrm{~cm}$ long tunnel right in front of the parenchymal bridge, $2 \mathrm{~cm}$ inferior to the porta hepatis. We have come across a similar case in a report of Ebby et al. [9]. They reported the sagittal fissure have become a complete tunnel having the round ligament in it with a missing quadrate lobe [8]. Our case shows a normal quadrate lobe as seen in Figure 3.

\section{CONCLUSIONS}

The origin, diameter, length, course and segmental distribution of the hepatic arteries are important for surgical/invasive approaches to the liver both in living donor and split liver transplantations. The present case with the segment IV supplied by both the rRHA and $\mathrm{MHA}$ is of great importance due to their possible implications in liver transplantations/surgeries. There is also a possibility to interrupt the blood supply to the liver when there is pancreaticoduodenectomy attempted to the patients with rRHA [15, 21, 22]. It should always be kept in mind that the origin of the hepatic artery and/or its branches may show many variations and should be under consideration beforehand the planned surgery. Also, using a multidetector computed tomography angiography can detect the rare hepatic arterial vascularisation [18].

The parenchymal variations like the one we have reported in this case should be kept in mind in terms of possible vascular variations associated their development.

\section{Acknowledgements}

We would like to commemorate the cadaver donor for this study. Also, we are grateful to our students, Dilara Unalan and Ali Eren Ozturk for their endless helps for taking the pictures and recording the video during our cadaver dissection.

\section{REFERENCES}

1. Alghamdi T, Viebahn $C$, Justinger $C$, et al. Arterial blood supply of liver segment IV and its possible surgical consequences. Am J Transplant. 2017; 17(4): 1064-1070, doi: 10.1111/ajt.14089, indexed in Pubmed: 27775870.

2. Allendorf JD, Bellemare S. Reconstruction of the replaced right hepatic artery at the time of pancreaticoduodenectomy. J Gastrointest Surg. 2009; 13(3): 555-557, doi: 10.1007/s11605-008-0578-8, indexed in Pubmed: 18642051.

3. Azoulay D, Castaing D, Adam R, et al. Transplantation of three adult patients with one cadaveric graft: wait or innovate. Liver Transpl. 2000; 6(2): 239-240, doi: 10.1002/ It.500060208, indexed in Pubmed: 10719027.

4. Beger O, Elvan Ö, Kurtoğlu Z. Replaced right hepatic artery and its segmental distribution. Anatomy. 2016; 9(3): 185-188, doi: 10.2399/ana.15.019.

5. Bismuth $\mathrm{H}$. Surgical anatomy and anatomical surgery of the liver. World J Surg. 1982; 6(1): 3-9, doi: 10.1007/ bf01656368.

6. Caliskan E, Acar T, Ozturk M, et al. Coeliac trunk and common hepatic artery variations in children: an analysis with computed tomography angiography. Folia Morphol. 2018; 77(4): 670-676, doi: 10.5603/FM.a2018.0037, indexed in Pubmed: 29651794.

7. Collardeau-Frachon S, Scoazec JY. Vascular development and differentiation during human liver organogenesis. Anat Rec (Hoboken). 2008; 291(6): 614-627, doi: 10.1002/ ar.20679, indexed in Pubmed: 18484606. 
8. Couinaud C. Le foie. Etudes anatomiques et chirurgicales. Masson, Paris 1957.

9. Ebby S, Ambike MV. Anatomical variation of ligamentum teres of liver: a case report. WebmedCentral ANATOMY. 2012; 3(5): WMC003389.

10. Ghosh SK. Variations in the origin of middle hepatic artery: a cadaveric study and implications for living donor liver transplantation. Anat Cell Biol. 2014; 47(3): 188-195, doi: 10.5115/acb.2014.47.3.188, indexed in Pubmed: 25276478.

11. Gurgacz AM, Horbaczewska A, Klimek-Piotrowska W, et al. Variations in hepatic vascularisation: lack of a proper hepatic artery. Two case reports. Folia Morphol. 2011; 70(2): 130-134, indexed in Pubmed: 21630235.

12. Hiatt JR, Gabbay J, Busuttil RW. Surgical anatomy of the hepatic arteries in 1000 cases. Ann Surg. 1994; 220(1): 50-52, indexed in Pubmed: 8024358.

13. Holbert BL, Baron RL, Dodd GD. Hepatic infarction caused by arterial insufficiency: spectrum and evolution of CT findings. AJR Am J Roentgenol. 1996; 166(4): 815-820, doi: 10.2214/ ajr.166.4.8610556, indexed in Pubmed: 8610556.

14. Jin GYu, Yu HC, Lim HS, et al. Anatomical variations of the origin of the segment 4 hepatic artery and their clinical implications. Liver Transpl. 2008; 14(8): 1180-1184, doi: 10.1002/lt.21494, indexed in Pubmed: 18668651.

15. Kim JiH, Gonzalez-Heredia R, Daskalaki D, et al. Totally replaced right hepatic artery in pancreaticoduodenectomy: is this anatomical condition a contraindication to minimally invasive surgery? HPB (Oxford). 2016; 18(7): 580-585, doi: 10.1016/j. hpb.2016.04.009, indexed in Pubmed: 27346138.
16. Michels NA. Newer anatomy of the liver and its variant blood supply and collateral circulation. Am J Surg. 1966; 112(3): 337-347, indexed in Pubmed: 5917302.

17. Onishi $H$, Kawarada $Y$, Das BC, et al. Surgical anatomy of the medial segment (S4) of the liver with special reference to bile ducts and vessels. Hepatogastroenterology. 2000; 47(31): 143-150, indexed in Pubmed: 10690598.

18. Skórzewska A, Stajgis P, Grzymisławska M, et al. Rare variations of hepatic arteries in association with variable origin of gastroduodenal artery found in multidetector computed tomography angiography. Folia Morphol. 2014; 73(4): 531-535, doi: 10.5603/FM.2014.0082, indexed in Pubmed: 25448917.

19. Sureka B, Mittal MK, Mittal A, et al. Variations of celiac axis, common hepatic artery and its branches in 600 patients. Indian J Radiol Imaging. 2013; 23(3): 223-233, doi: 10.4103/0971-3026.120273, indexed in Pubmed: 24347852.

20. Suzuki H. [Correlation and anomalies of the vascular structure in Glisson's area around the hepatic hilum, from the standpoint of hepatobiliary surgery]. Nihon Geka Hokan. 1982; 51(5): 713-731, indexed in Pubmed: 7159135.

21. Wang $S, H e X$, Li Z, et al. Characterization of the middle hepatic artery and its relevance to living donor liver transplantation. Liver Transpl. 2010; 16(6): 736-741, doi: 10.1002/lt.22082, indexed in Pubmed: 20517907.

22. Yang SH, Yin YHu, Jang JY, et al. Assessment of hepatic arterial anatomy in keeping with preservation of the vasculature while performing pancreatoduodenectomy: an opinion. World J Surg. 2007; 31(12): 2384-2391, doi: 10.1007/ s00268-007-9246-5, indexed in Pubmed: 17922256. 